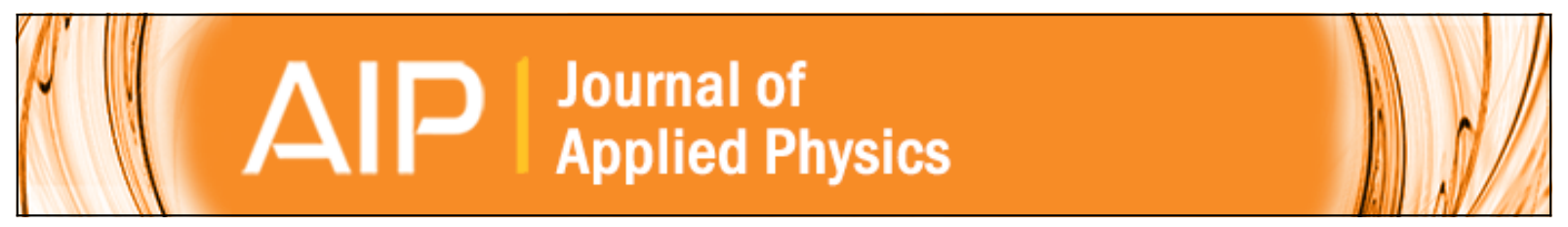

\title{
Iron detection in crystalline silicon by carrier lifetime measurements for arbitrary injection and doping
}

D. H. Macdonald, L. J. Geerligs, and A. Azzizi

Citation: Journal of Applied Physics 95, 1021 (2004); doi: 10.1063/1.1637136

View online: http://dx.doi.org/10.1063/1.1637136

View Table of Contents: http://scitation.aip.org/content/aip/journal/jap/95/3?ver=pdfcov

Published by the AIP Publishing

\section{Articles you may be interested in}

Temperature dependent carrier lifetime studies on Ti-doped multicrystalline silicon

J. Appl. Phys. 105, 124510 (2009); 10.1063/1.3139286

Doping dependence of the carrier lifetime crossover point upon dissociation of iron-boron pairs in crystalline silicon

Appl. Phys. Lett. 89, 142107 (2006); 10.1063/1.2358126

Effect of gettered iron on recombination in diffused regions of crystalline silicon wafers

Appl. Phys. Lett. 88, 092105 (2006); 10.1063/1.2181199

Electronic properties of iron-boron pairs in crystalline silicon by temperature- and injection-level-dependent lifetime measurements

J. Appl. Phys. 97, 103708 (2005); 10.1063/1.1897489

Iron detection in polished and epitaxial silicon wafers using generation lifetime measurements

J. Appl. Phys. 81, 7345 (1997); 10.1063/1.365440

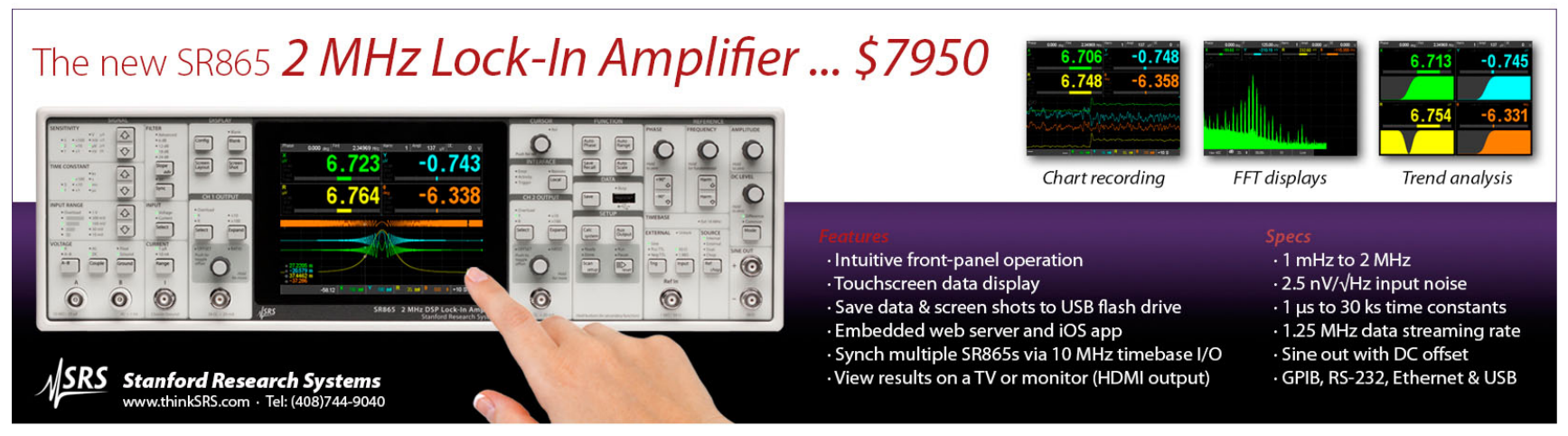




\title{
Iron detection in crystalline silicon by carrier lifetime measurements for arbitrary injection and doping
}

\author{
D. H. Macdonald, L. J. Geerligs, and A. Azzizi \\ ECN Solar Energy, PO Box 1, NL-1755 ZG Petten, The Netherlands
}

(Received 14 July 2003; accepted 6 November 2003)

\begin{abstract}
An existing technique for accurate measurement of iron in silicon, which was previously restricted to low injection and a narrow doping range, has been extended to arbitrary injection and doping levels. This allows contactless lifetime measurement techniques to be used for very sensitive and rapid iron detection under a wide range of conditions. In addition, an easily measured and unambiguous "fingerprint" of iron in silicon has been identified. It is based on the invariant nature of the excess carrier density at which the injection-dependent lifetime curves, measured before and after iron-boron pair dissociation, cross over. This characteristic crossover point lies in the narrow range of 1.4 to $2.0 \times 10^{14} \mathrm{~cm}^{-3}$, provided only that the boron concentration is below 5 $\times 10^{16} \mathrm{~cm}^{-3}$. To demonstrate the value of these techniques, they have been applied to photovoltaic-grade cast multicrystalline silicon wafers. (C) 2004 American Institute of Physics.
\end{abstract}

[DOI: $10.1063 / 1.1637136]$

\section{INTRODUCTION}

The presence of interstitial iron $\left(\mathrm{Fe}_{i}\right)$ in silicon can dramatically reduce the nonequilibrium carrier lifetime, even at very low concentrations. It is therefore not surprising that the most sensitive technique available for detecting iron contamination is based on lifetime or diffusion length measurements. This widely used technique was developed by Zoth and Bergholz, ${ }^{1}$ and utilizes the fact that the iron content of a boron-doped $p$-type $\mathrm{Si}$ wafer can be deliberately cycled between being mostly present as either $\mathrm{Fe}_{i}$, or as $\mathrm{FeB}$ pairs. Courtesy of their different energy levels and capture cross sections, these two forms of iron have markedly different recombination properties. This leads to significant changes in diffusion length after dissociating the FeB pairs, either by thermal annealing or optical activation.

Zoth and Bergholz showed that, by measuring the lowinjection diffusion length before $\left(L_{0}\right)$ and after $\left(L_{1}\right)$ pair dissociation, and assuming that all other recombination processes remain unchanged, the total iron concentration $\left(\mathrm{cm}^{-3}\right)$ can be found by

$$
[\mathrm{Fe}]=A\left(\frac{1}{L_{1}^{2}}-\frac{1}{L_{0}^{2}}\right)=C\left(\frac{1}{\tau_{1}}-\frac{1}{\tau_{0}}\right)
$$

The prefactor $A$ was determined empirically to be 1.06 $\times 10^{16} \mu \mathrm{m}^{2} \mathrm{~cm}^{-3}$ for silicon wafers of dopant density $1-3$ $\times 10^{15} \mathrm{~cm}^{-3}$ (resistivity range from 5 to $15 \Omega \mathrm{cm}$ ). If the low-injection carrier lifetime $\tau$ is measured rather than the diffusion length, then the prefactor $C$ is related to $A$ via $A$ $=D_{n} C$, where $D_{n}$ is the minority carrier diffusion coefficient.

Equation (1) has become widely used in commercial applications for contamination control in microelectronics, as well as being an extremely useful tool for researching iron in silicon. $^{2-4}$ It is most sensitive when iron is the dominant recombination center, but is also valid when other centers are present as well provided they are not affected by the dissociation procedure.

However, there are some restrictions on the use of this specific technique. Firstly, the diffusion lengths must be measured under low-injection conditions. The most reliable technique for this is the surface photovoltage method (SPV), since it operates in true low injection. However, other lifetime measurement techniques, such as microwave-detected photoconductance decay or the quasi-steady-state photoconductance $(\mathrm{QSSPC})^{5}$ technique, suffer from reduced sensitivity at low-injection. Equally importantly, they are also affected by minority carrier trapping artifacts at low injection. ${ }^{6}$ Such trapping causes a large excess of majority carriers, which distorts the photoconductance, since it comprises both minority and majority carriers. Voltage-based techniques such as SPV are not affected by trapping because they detect only minority carriers. As a result of these considerations, the photoconductance-based lifetime techniques, which are widely used and otherwise more convenient than SPV, generally operate at mid to high injection. In this regime, the value of $C$ will be different (often very significantly) than that determined for SPV measurements.

Secondly, even for low-injection SPV measurements, if the dopant density is well outside the range from 1 to 3 $\times 10^{15} \mathrm{~cm}^{-3}$, it is not entirely valid to use the prefactor found by Zoth and Bergholz. This is due to the particular properties of $\mathrm{Fe}_{i}$ and $\mathrm{FeB}$ : the energy level of the $\mathrm{FeB}$ center is relatively shallow, and its impact on the low-injection lifetime therefore depends on the dopant density. On the other hand, $\mathrm{Fe}_{i}$, being a deep center, produces a low-injection lifetime that is independent of the doping level. ${ }^{7}$ Since the prefactor $C$ is determined by the difference of the inverse lifetimes, it must also vary with the dopant density.

In this article, we make use of the known recombination parameters of both $\mathrm{FeB}$ pairs and $\mathrm{Fe}_{i}$ to determine the prefactor $C$ for arbitrary dopant density and injection level. This 
allows the current $\mathrm{Fe}$ detection method to be greatly extended. As an example, the Fe concentration in solar grade multicrystalline $(\mathrm{mc})$ silicon $(1 \Omega \mathrm{cm})$ is measured before and after gettering, using the QSSPC technique operating at excess carrier densities in the range from $10^{14}$ to $10^{16} \mathrm{~cm}^{-3}$. Despite the quite strong injection dependence of the measured lifetimes in this range, the calculated $\mathrm{Fe}$ concentration is shown to be independent of the injection level, as indeed it should be.

An important aspect of using this technique is that the proportion of $\mathrm{Fe}$ present as $\mathrm{FeB}$ pairs must be known reasonably accurately during the measurements before and after dissociation. By comparing optical and thermal dissociation, we demonstrate that strong optical illumination $(>0.1$ $\mathrm{W} \mathrm{cm}^{-2}$ ) results in almost complete $(>99 \%)$ dissociation of the FeB pairs. Allowing a sample to relax in the dark for at least $24 \mathrm{~h}$ results in almost complete re-pairing for typical dopant densities.

In addition, a distinctive fingerprint of Fe contamination in silicon is presented. It is based on measuring the injection level at which the lifetime curves before and after FeB pair dissociation crossover (cr). The fact that such a crossover exists has been observed previously. ${ }^{8-10}$ However, in this article, we show this crossover point is independent of the dopant density. Measuring the excess carrier density at which the crossover occurs allows fast and unambiguous identification of iron in silicon.

\section{DETERMINING THE FACTOR $C$ FOR ARBITRARY INJECTION AND DOPING}

When the density of a given type of recombination center is small, it is possible to use Shockley-Read-Hall (SRH) statistics ${ }^{11,12}$ to model their impact on the excess carrier lifetime. Due to its relatively low solubility at typical processing temperatures, this is almost always true for iron in silicon. For $p$-type silicon with an acceptor concentration $N_{A}$, the SRH lifetime $\tau_{S R H}$ is given by ${ }^{13}$

$$
\tau_{\mathrm{SRH}}=\frac{\tau_{p 0}\left(n_{1}+\Delta n\right)+\tau_{n 0}\left(N_{A}+p_{1}+\Delta n\right)}{N_{A}+\Delta n} .
$$

The parameters $\tau_{n 0}$ and $\tau_{p 0}$ are related to the electron and hole capture cross sections $\sigma_{n}$ and $\sigma_{p}$ of the recombination center, their density $N$, and the carrier thermal velocities $v_{\text {th }}$ :

$$
\tau_{n 0}=\frac{1}{N \sigma_{n} v_{\text {th }}} \quad \tau_{p 0}=\frac{1}{N \sigma_{p} v_{\text {th }}} .
$$

The factors $n_{1}$ and $p_{1}$ are the equilibrium carrier densities when the recombination center energy $E_{T}$ coincides with the Fermi level. The thermal velocity ${ }^{14}$ is taken as 1.1 $\times 10^{7} \mathrm{~cm} \mathrm{~s}^{-1}$.

When there are two types of recombination center present, such as for boron-doped $p$-type silicon containing both $\mathrm{FeB}$ and $\mathrm{Fe}_{\mathrm{i}}$, their combined impact, together with the impact of other recombination processes, results in an effective lifetime
TABLE I. Recombination parameters of $\mathrm{FeB}$ and $\mathrm{Fe}_{i}$ used in the calculation of $C$.

\begin{tabular}{llll}
\hline \hline & $E_{T}(\mathrm{eV})$ & $\sigma_{n}\left(\mathrm{~cm}^{-2}\right)$ & $\sigma_{p}\left(\mathrm{~cm}^{-2}\right)$ \\
\hline $\mathrm{Fe}_{i}$ & $E_{V}+0.38$ & $5 \times 10^{-14}$ & $7 \times 10^{-17}$ \\
$\mathrm{FeB}$ & $E_{C}-0.23$ & $3 \times 10^{-14}$ & $2 \times 10^{-15}$ \\
\hline \hline
\end{tabular}

Note. Data taken from Refs. 7 and 15.

$$
\frac{1}{\tau_{\text {eff }}}=\frac{1}{\tau_{\mathrm{FeB}}}+\frac{1}{\tau_{\mathrm{Fe}_{i}}}+\frac{1}{\tau_{\text {other }}} .
$$

The robustness of the technique pioneered by Zoth and Bergholz is largely due to the use of the difference of the inverse effective lifetime before and after dissociating the FeB pairs. Hence, the term involving other processes cancels out, provided these processes are not altered by the dissociation step. In addition since Zoth and Bergholz used SPV for diffusion length measurements, they were naturally restricted to the low-injection regime, in which the lifetimes are independent of the excess carrier density.

However, in general, the value of the constant $C$ in Eq. (1) does depend on both the dopant density and the excess carrier density. It will also depend on the precise proportion of $\mathrm{Fe}$ present as $\mathrm{FeB}$ pairs and $\mathrm{Fe}_{i}$ before and after the dissociation process. In the simplest case, we may assume that $100 \%$ of the $\mathrm{Fe}$ is paired before dissociation and $100 \%$ interstitial afterwards, an assumption which is shown in later sections to be reasonable when strong optical dissociation is used. By using known values of the energy levels and capture cross sections, ${ }^{7,15}$ shown in Table I, $C$ can be calculated directly for any dopant density or excess carrier density by substituting Eq. (2) for both $\mathrm{Fe}_{i}$ and $\mathrm{FeB}$ into Eq. (1). In other cases, in which either association or dissociation is incomplete, the value of $C$ can be calculated with the aid of Eq. (4).

As an example, Fig. 1 shows calculated values of $1 / C$ as a function of $\Delta n$ for a range of dopant densities. In these plots, complete pairing and dissociation are assumed. At very low injection conditions, the value of $C$ is always positive (meaning lifetimes decrease after dissociation) and indepen-

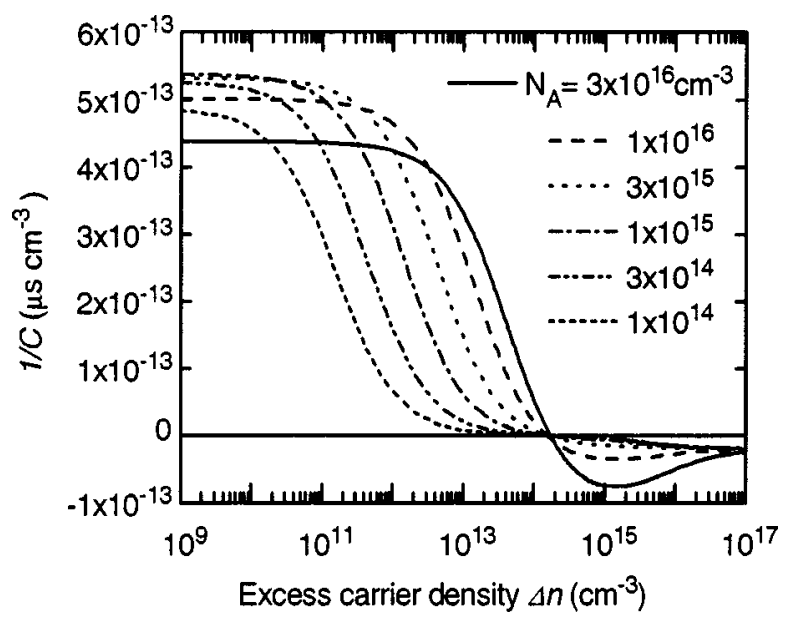

FIG. 1. Inverse lifetime prefactor $1 / C$ versus the excess carrier density $\Delta n$ for various dopant densities. 


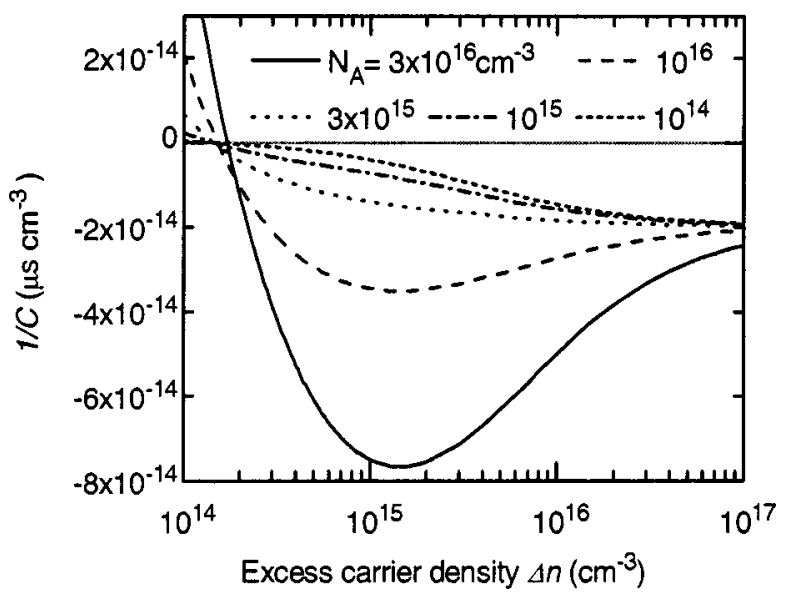

FIG. 2. Inverse lifetime prefactor $1 / C$ versus the excess carrier density $\Delta n$ for various dopant densities, for moderate $\Delta n$.

dent of the excess carrier concentration. This is the region in which Zoth and Bergholz's SPV technique is valid. Figure 1 shows that even when low-injection conditions prevail, the value of $C$ does indeed vary with the dopant density. This variation becomes even greater when considering the corresponding diffusion length constant $A$, due to the doping dependence of $D_{n}$.

At injection levels greater than approximately $\Delta n=2$ $\times 10^{14} \mathrm{~cm}^{-3}$, the value of $C$ becomes negative for all dopant densities, meaning that the lifetime increases after dissociation. It is this region, as magnified in Fig. 2, which is of relevance for determining Fe concentrations by lifetime techniques that operate under medium to high injection.

In order to minimize the uncertainties when using this approach for Fe detection, it is desirable to measure the change in lifetime at a fixed value of $\Delta n$ near which $C$ does not change too rapidly. For commonly used dopant densities this corresponds to the region $\Delta n=10^{15}-10^{16} \mathrm{~cm}^{-3}$. Above this range, the presence of Auger or emitter recombination may cap the magnitude of the effective lifetime, therefore reducing sensitivity.

The detection limit of the technique will depend on the value of $C$, and this of course varies with injection level. The low-injection SPV technique has been shown capable of detecting Fe concentrations as low as $7 \times 10^{7} \mathrm{~cm}^{-3}$ in $1-10$ $\Omega \mathrm{cm}$ material, ${ }^{2}$ in which case the diffusion length changed very slightly from 1126 to $1119 \mu \mathrm{m}$ after dissociation. These measurements required a 3-mm-thick Si slab to avoid excessive rear surface recombination, and averaging of several measurements to reduce noise. Using other lifetime techniques at an injection level of $1 \times 10^{15} \mathrm{~cm}^{-3}$, and assuming excellent surface passivation capping the effective lifetime to $5000 \mu \mathrm{s}$, the corresponding change in lifetime would be from around 4990 to $4950 \mu$ s for $7 \times 10^{7} \mathrm{~cm}^{-3}$ of Fe. This means a relative uncertainty of less than $1 \%$ in the lifetime measurements would be required, which would only be possible with extremely stable surface passivation and averaging. However, using mid- to high-injection measurements, $\mathrm{Fe}$ detection well below $10^{10} \mathrm{~cm}^{-3}$ is certainly feasible.

A verification of the calculated values can be obtained by comparison with the empirical value of $A=1.06$

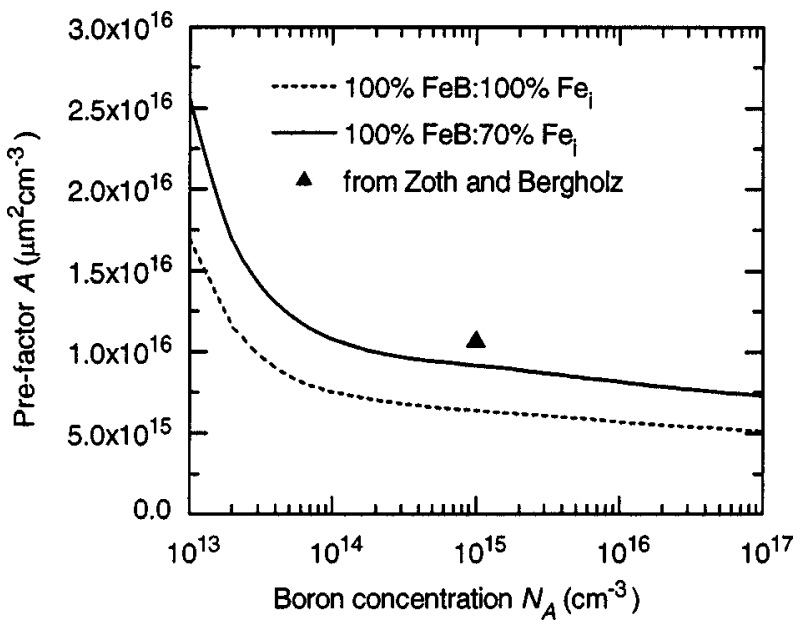

FIG. 3. Comparison of the diffusion length prefactor A from Zoth and Bergholz with those calculated from the recombination parameters of Table I. The case of $100 \% \mathrm{FeB}$ before and $100 \% \mathrm{Fe}_{i}$ after dissociation corresponds to strong optical dissociation, while the $100 \%: 70 \%$ case corresponds to the thermal dissociation used by Zoth and Bergholz.

$\times 10^{16} \mu \mathrm{m}^{2} \mathrm{~cm}^{-3}$ obtained for 5-15 $\Omega \mathrm{cm}$ material by Zoth and Bergholz. They used thermal dissociation, and estimated that almost $100 \%$ of the iron was paired before thermal treatment, and $70 \%$ dissociated after heating and then quenching on an Al plate.

Figure 3 shows the values of $A$ calculated from the SRH model for a range of dopant densities. Two cases are shown: the first assuming 70\% dissociation as suggested by Zoth and Bergholz, and the second with $100 \%$ dissociation (relevant for strong optical dissociation). The electron diffusion coefficient $D_{n}$ was determined from PC1D version 5.5. ${ }^{16}$ The equilibrium proportion present as FeB pairs before dissociation was determined for each resistivity according to the empirical relation $^{17}$

$$
\frac{[\mathrm{FeB}]}{\left[\mathrm{Fe}_{i}\right]}=10^{-23} N_{A} \exp \left(\frac{0.65}{k T}\right)
$$

For a dopant density of $N_{A}=1.0 \times 10^{16} \mathrm{~cm}^{-3}$, as is typical for photovoltaic silicon, this results in $99.99 \%$ as FeB pairs at $300 \mathrm{~K}$. Even when the dopant density is as low as $1 \times 10^{14} \mathrm{~cm}^{-3}$, this value only decreases to $99 \%$.

Figure 3 shows that there is good agreement with Zoth and Bergholz's single data point, giving confidence in the methodology used here. This approach allows the correct prefactor $A$ to be determined for SPV measurements over a large range of resistivity.

For optical dissociation, the 100:100 curve should be quite accurate, as shown in Sec. IV, assuming the postdissociation measurement is performed quickly enough to avoid re-pairing. However, the situation is more complex for thermal dissociation, since at the elevated temperatures that occur during quenching, the time constant for re-pairing may be very short, especially for more highly doped samples (see Sec. IV). Therefore, the $70 \%$ assumed by Zoth and Bergholz would most likely not hold for significantly different doping levels when using the same quenching conditions. For 
photovoltaic-grade silicon, which is usually around $1 \Omega \mathrm{cm}$ in resistivity, it is preferable to use optical dissociation to avoid these uncertainties.

\section{THE FIXED CROSSOVER POINT}

It should always be kept in mind that there are numerous possible explanations for an observed change in carrier lifetime after exposure to illumination. For example, the surface recombination velocity of certain types of plasma-enhanced chemical vapor deposited (PECVD) silicon nitride films is thought to be altered by the capture of light-induced carriers at so-called K-centers. ${ }^{18}$ Another well-known example is the carrier-induced degradation associated with metastable boron-oxygen complexes in Czochralski silicon, ${ }^{19}$ which causes a reduction in lifetime across all injection levels. The breaking of $\mathrm{Cu}-\mathrm{Cu}$ pairs by illumination is also believed to reduce the lifetime. ${ }^{20}$ It is therefore important to have an independent means of checking if any observed changes are actually due to the presence of iron.

One such method, suggested by Zoth and Bergholz, is to measure the characteristic re-pairing time as the lifetime relaxes to equilibrium conditions at RT. This approach, as discussed in Sec. IV, indeed provides convincing evidence for the presence of $\mathrm{Fe}$, but has the disadvantage of being somewhat time consuming.

Courtesy of the extreme asymmetry between the electron and hole capture cross section of $\mathrm{Fe}_{i}$, it turns out that there is another robust and convenient "fingerprint" for Fe in silicon. For all dopant densities in Figs. 1 and 2, there is a particular excess carrier density $\Delta n_{\text {cr }}$ at which the lifetime remains unchanged after dissociation. Despite the dopant density changing by more than two orders of magnitude in these figures, the value of this crossover point remains almost constant at around $1.5 \times 10^{14} \mathrm{~cm}^{-3}$. It is also possible to show that the value of this crossover point is independent of the relative populations before and after dissociation, although of course greater dissociation will allow more accurate determination of $\Delta n_{\mathrm{cr}}$. The constancy of this crossover point makes it an excellent identifier of Fe.

In order to demonstrate these useful properties, consider the conditions under which the lifetimes before and after dissociation are equal:

$$
\frac{1}{\tau_{\mathrm{FeB}}\left(N_{1}\right)}+\frac{1}{\tau_{\mathrm{Fe}_{i}}\left(N_{2}\right)}=\frac{1}{\tau_{\mathrm{FeB}}\left(N_{3}\right)}+\frac{1}{\tau_{\mathrm{Fe}_{i}}\left(N_{4}\right)} .
$$

In this expression, the symbols $N_{1}$, etc. represent the density of recombination centers, such that $N_{1}+N_{2}=N_{3}+N_{4}$ $=\left[\mathrm{Fe}_{i}\right]+[\mathrm{FeB}]=[\mathrm{Fe}]_{\text {total }}$. The impact of other recombination mechanisms, which are not altered by the dissociation process, cancel out and are not shown.

In Appendix A, it is shown that Eq. (6) is equivalent to stating that

$$
\tau_{\mathrm{FeB}}\left(N_{1}\right)=\tau_{\mathrm{Fe}_{i}}\left(N_{1}\right) .
$$

In other words, the crossover point will be the excess carrier density at which the lifetimes due to any density $N$ of FeB pairs equals the lifetime due to the same density of $\mathrm{Fe}_{\mathrm{i}}$.
TABLE II. Values of the crossover point from the literature and this work for samples of various dopant densities and growth techniques. The values from Ref. 10 are shown as upper limits, since they reported the peak excess carrier concentration, rather than the average.

\begin{tabular}{crlrc}
\hline \hline $\begin{array}{c}\text { Resist. } \\
(\Omega \mathrm{cm})\end{array}$ & $N_{A}\left(\mathrm{~cm}^{-3}\right)$ & Growth & $\begin{array}{c}\text { Crossover } \\
\text { point }\left(\mathrm{cm}^{-3}\right)\end{array}$ & Ref. \\
\hline 0.3 & $6.6 \times 10^{16}$ & $\mathrm{FZ}$ & $1.2 \times 10^{14}$ & 7 \\
1 & $1.7 \times 10^{16}$ & $\mathrm{FZ}$ & $1.4 \times 10^{14}$ & 7 \\
5 & $3 \times 10^{15}$ & $\mathrm{FZ}$ & $2.5 \times 10^{14}$ & 7 \\
$8-15$ & $1.0-1.7 \times 10^{15}$ & $\mathrm{Cz}$ & $1-2 \times 10^{14}$ & 8 \\
20 & $7 \times 10^{14}$ & $\mathrm{Cz}$ & $<2.7 \times 10^{14}$ & 10 \\
50 & $3 \times 10^{14}$ & $\mathrm{Cz}$ & $<3.6 \times 10^{14}$ & 10 \\
0.7 & $2.2 \times 10^{16}$ & $\mathrm{mc}-\mathrm{Si}$ & $2.0 \times 10^{14}$ & This work \\
1.1 & $1.5 \times 10^{16}$ & $\mathrm{mc}-\mathrm{Si}$ & $1.3 \times 10^{14}$ & This work \\
\hline \hline
\end{tabular}

We may then use the SRH model [Eq. (2)] to solve this expression for $\Delta n_{\mathrm{cr}}$. Due to the extremely small hole cross section of $\mathrm{Fe}_{i}$, this reduces to

$$
\Delta n_{\mathrm{cr}} \approx \frac{\tau_{p 0, \mathrm{FeB}}}{\tau_{p 0, \mathrm{Fe}_{i}}} n_{1, \mathrm{FeB}}=\frac{\sigma_{p, \mathrm{Fe}_{i}}}{\sigma_{p, \mathrm{FeB}}} n_{1, \mathrm{FeB}}=1.4 \times 10^{14} \mathrm{~cm}^{-3},
$$

provided that

$$
N_{A} \ll\left|\frac{\tau_{p 0, \mathrm{FeB}} n_{1, \mathrm{FeB}}}{\tau_{n 0, \mathrm{Fe}_{i}}-\tau_{n 0, \mathrm{FeB}}}\right|=1.5 \times 10^{17} \mathrm{~cm}^{-3} .
$$

As $N_{A}$ approaches the value on the right of Eq. (9), the crossover point begins to increase. However, so long as $N_{A}$ $<5 \times 10^{16} \mathrm{~cm}^{-3}$, then $\Delta n_{\text {cr }}$ will remain below $2.0 \times 10^{14}$. For most practical purposes, therefore, the crossover point resides in the narrow range from 1.4 to $2.0 \times 10^{14} \mathrm{~cm}^{-3}$.

There do exist some published values of the crossover point in the literature, ${ }^{7,8,10}$ which are shown in Table II. Results from Sec. IV on photovoltaic-grade mc-silicon are also included. Together, they provide excellent support for the value just calculated from the energy levels and capture cross sections. They also confirm that it is independent of the dopant density or silicon growth technique (i.e., presence of other recombination centers).

\section{IRON IN SOLAR-GRADE mC-SILICON: AN EXAMPLE}

Boron-doped multicrystalline silicon is in common use in photovoltaics, offering a reasonable compromise between economic cost and electronic quality. It often contains significant amounts of interstitial $\mathrm{Fe}$, as shown in recent studies, ${ }^{9}$ as well as other metals, ${ }^{21}$ and so provides a suitable test for the techniques developed here. Due to its detrimental impact on lifetime, and therefore solar cell efficiency, it is also of direct interest to be able to identify Fe in such material with certainty, and to determine its concentration.

\section{A. Identification by the crossover point}

Figure 4 shows injection-dependent lifetime measurements on a commercially produced, cast mc-silicon wafer with $N_{A}=1.5 \times 10^{16} \mathrm{~cm}^{-3}$. Lifetimes were measured using the QSSPC technique, with the surfaces passivated by PECVD silicon nitride. ${ }^{22}$ Measurements were taken after 24 


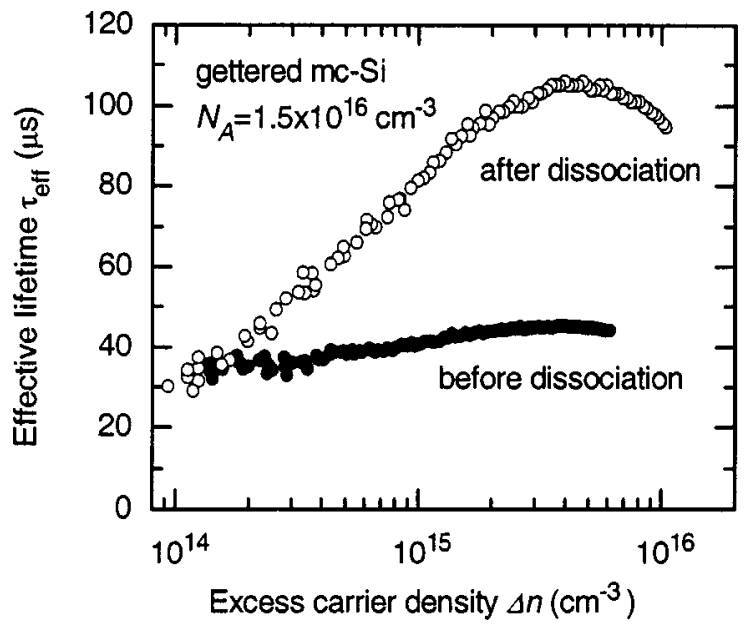

FIG. 4. Effective lifetime versus excess carrier concentration for a partially gettered mc-Si sample containing Fe, showing the crossover of the lifetime curves at $1.3 \times 10^{14} \mathrm{~cm}^{-3}$ before and after optical dissociation.

$\mathrm{h}$ in the dark and again after 5 min exposure to $0.1 \mathrm{~W} \mathrm{~cm}^{-2}$ white light from a halogen lamp (approximately equivalent to 1 sun). The crossover point at $1.3 \times 10^{14} \mathrm{~cm}^{-3}$ corresponds very well with that expected from the SRH model. A second wafer from another manufacturer, with $N_{A}=2.2$ $\times 10^{16} \mathrm{~cm}^{-3}$, was similarly measured and found to have a crossover occurring at $2 \times 10^{14} \mathrm{~cm}^{-3}$.

The two wafers had previously received on one side a belt furnace phosphorus diffusion, resulting in a sheet resistivity of around $50 \Omega /$ square. The diffused layer, which was removed before surface passivation, provided some partial gettering of $\mathrm{Fe}$ and other impurities. In neighboring nongettered wafers, the presence of minority carrier trapping centers ${ }^{6}$ obscured the lifetime measurements below approximately $2 \times 10^{14} \mathrm{~cm}^{-3}$. The use of a correction procedure for subtracting the underlying excess photoconductance caused by traps ${ }^{23}$ allows the crossover point to be observed in such cases. However, if the trap density is much higher than 5 $\times 10^{14} \mathrm{~cm}^{-3}$, this procedure becomes too uncertain at the crossover point. In such cases, this technique cannot be used to confirm the presence of iron in silicon.

\section{B. Identification by re-pairing kinetics}

To support the assertion that the light-induced changes in the multicrystalline samples above are due to $\mathrm{Fe}$, the repairing rate at RT was measured and compared with previously reported values. This approach was recently used by Ballif et al. ${ }^{9}$ to identify $\mathrm{Fe}$ in cast mc-silicon.

After the FeB concentration has been perturbed from its equilibrium value, either by optical or thermal dissociation, the resulting excess $\mathrm{Fe}_{i}$ re-associates according to a monoexponential decay with a characteristic time $\tau_{\text {assoc }}$. This parameter is well known and depends on both the dopant density and temperature: ${ }^{1}$

$$
\tau_{\text {assoc }}=\frac{4.3 \times 10^{5}}{N_{A}} T \exp \left(\frac{0.68}{k T}\right) .
$$

If the lifetime is measured at a carrier density above the crossover point, this repairing will result in a gradual $d e-$

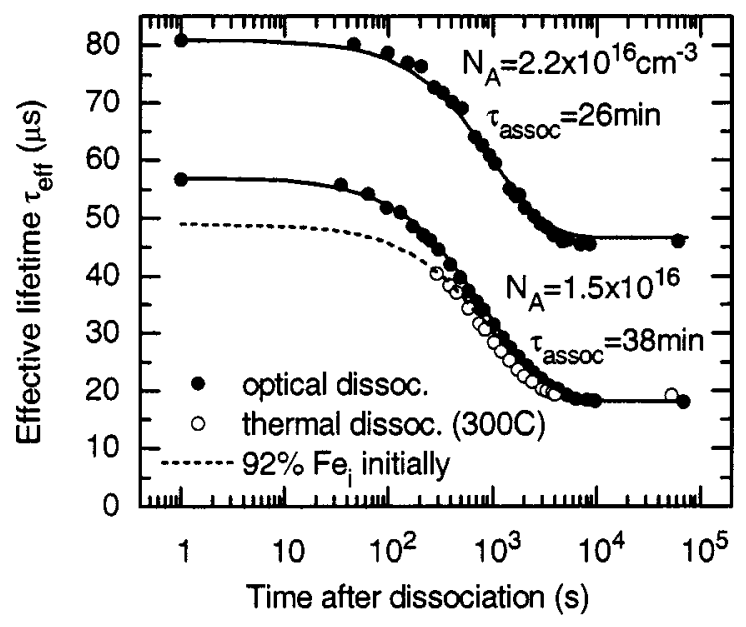

FIG. 5. Decrease of the effective lifetime, measured at $\Delta n=3$ $\times 10^{15} \mathrm{~cm}^{-3}$, of nongettered mc-Si samples as the FeB pairs re-form after optical or thermal $\left(300{ }^{\circ} \mathrm{C}\right.$ then quenched in water) dissociation. The lines show mono-exponential fits with time constants determined from Eq. (10). For the thermal dissociation case, the proportion as $\mathrm{Fe}_{i}$ immediately after quenching is $92 \%$.

crease in the lifetime. Figure 5 shows a measurement of such relaxation for a nongettered neighboring wafer of the multicrystalline sample of Fig. 4, as well as another slightly more heavily doped sample. The lifetimes were measured at $\Delta n$ $=3 \times 10^{15} \mathrm{~cm}^{-3}$. Dissociation was achieved by exposing the samples to white light of $0.1 \mathrm{~W} \mathrm{~cm}^{-2}$ intensity for $5 \mathrm{~min}$. The decay of the lifetime as the $\mathrm{FeB}$ pair concentration approaches equilibrium fits very well with a single exponential model with $\tau_{\text {assoc }}=38$ and $26 \mathrm{~min}$, as calculated from Eq. (10), for these two wafers with $N_{A}=1.5 \times 10^{16}$ and 2.2 $\times 10^{16} \mathrm{~cm}^{-3}$, respectively. Hence, there is little doubt that the observed changes in lifetime are due to Fe.

We may now turn our attention to calculating the $\mathrm{Fe}$ concentration from the change in lifetime at a given excess carrier density. This requires a reasonable knowledge of what proportion of $\mathrm{Fe}$ is paired before and after dissociation, whether by optical or thermal means, so that an appropriate value of $C$ can be determined.

\section{Paired fraction before dissociation}

We may use Eq. (5) to determine the equilibrium fraction of FeB pairs before dissociation. As discussed carlier for most dopant densities this is well above 99\% at RT. However, the time required for a sample to reach equilibrium at RT depends inversely on the dopant density [Eq. (10)]. With $N_{A}=1.0 \times 10^{16} \mathrm{~cm}^{-3}$, as is representative of the wafers used in this study, this leads to a value of $57 \mathrm{~min}$ at $300 \mathrm{~K}$. Hence after only $5 \mathrm{~h}$, the density of FeB pairs will reach $99 \%$ of its equilibrium value (which is $99.99 \%$ of the total Fe concentration for this dopant density). In this study, wafers were rested in the dark for at least $12 \mathrm{~h}$ before measurement, ensuring almost complete pairing. If using more lightly doped material, however, the required relaxation times at RT are much longer: 50 and $500 \mathrm{~h}$ for $N_{A}=1.0 \times 10^{15}$ and 1.0 $\times 10^{14} \mathrm{~cm}^{-3}$, respectively.

It is also possible that the measurement process itself causes significant dissociation of FeB pairs. For SPV, this is 


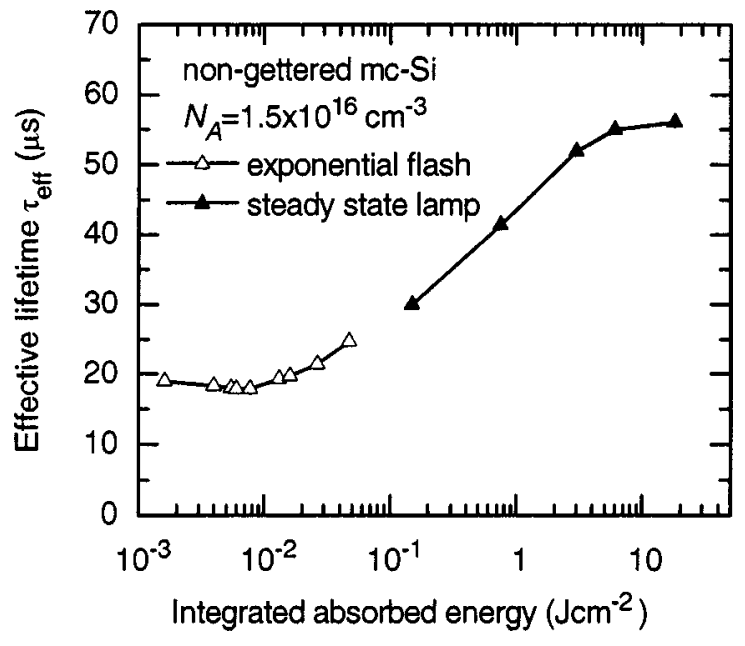

FIG. 6. Effective lifetime of a nongettered mc-Si wafer versus integrated absorbed energy from either an exponential flash or a steady-state lamp. The point at which the lifetime begins to increase marks the beginning of $\mathrm{FeB}$ pair dissociation.

unlikely since the illumination used to generate the photovoltage is very weak. For other mid- and high-injection techniques, much more intense illumination is generally used. The QSSPC technique, as used in this work, requires flashing the sample with a xenon lamp with an exponential decay time of $1.9 \mathrm{~ms}$. The peak intensity can be varied using gray filters, and ranges between 0.01 and $25 \mathrm{~W} \mathrm{~cm}^{-2}$.

To check the extent of dissociation caused by this illumination, the multicrystalline wafer containing Fe with $N_{A}$ $=1.5 \times 10^{16} \mathrm{~cm}^{-3}$ from Fig. 5 was allowed to relax fully overnight. The lifetime was measured by QSSPC with flashes of increasing intensity, and then also after steadystate illumination of $0.1 \mathrm{~W} \mathrm{~cm}^{-2}$ for increasing lengths of time. The measured lifetimes are shown in Fig. 6 as a function of the integrated absorbed energy. The results show that if the absorbed energy is kept below $0.01 \mathrm{~J} \mathrm{~cm}^{-2}$, dissociation is avoided. This corresponds to a peak intensity of around $5 \mathrm{~W} \mathrm{~cm}^{-2}$ for our flash, a value that was not exceeded during any measurements presented in this work. It should be noted, however, that if the carrier lifetime is much larger than in the samples used here, the threshold intensity would be reduced due to the higher carrier densities, and hence greater dissociation rate, at a given illumination level.

It seems, then, that the assumption of almost complete pairing during the initial measurement is reasonable, at least in the cases presented here. The final question then is what proportion of iron is interstitial during the second measurement, after optical dissociation.

\section{Paired fraction after dissociation}

One approach to answering this is to compare the effects of optical and thermal dissociation. The advantage of thermal dissociation is that the fraction of FeB pairs is easily determined from Eq. (5). However, it is important to be aware of possible re-pairing during cooling. To minimize the extent of this re-pairing, we used a vertical quench in water from between two heated metal plates.

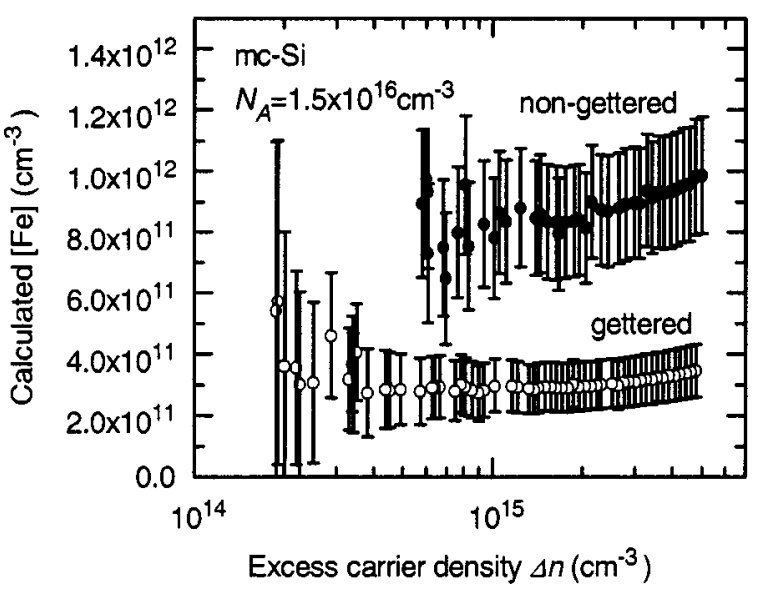

FIG. 7. Calculated Fe concentration $[\mathrm{Fe}]_{\text {total }}$ for the nongettered and partially gettered neighboring mc-Si samples, revealing that for each sample $[\mathrm{Fe}]_{\text {total }}$ is independent of $\Delta n$ within measurement uncertainty.

The choice of anneal temperature is important, because at higher temperatures the dissociation is more complete, but the re-pairing time is faster. The best approach is to use a relatively high temperature at which the dissociation approaches saturation. Any slight cooling before the sample hits the quench solution will then have less impact on the final interstitial proportion.

To illustrate this point, according to Eq. (5), annealing at $300{ }^{\circ} \mathrm{C}$ results in $92 \%$ present as $\mathrm{Fe}_{i}$ in silicon with $N_{A}$ $=1.5 \times 10^{16} \mathrm{~cm}^{-3}$. However, for this doping and temperature, the characteristic re-pairing time is only $0.02 \mathrm{~s}$. This is not so important, however, because even if the sample cools relatively slowly to $280{ }^{\circ} \mathrm{C}$ before quenching, the proportion as $\mathrm{Fe}_{i}$ is still $89 \%$.

By contrast, at 200 and $180^{\circ} \mathrm{C}$, the equilibrium proportions as $\mathrm{Fe}_{i}$ are $44 \%$ and $22 \%$, respectively, meaning a much more significant loss of $\mathrm{Fe}_{i}$ is possible if the quenching is not perfect.

The results of such annealing at $300^{\circ} \mathrm{C}$ are shown in Fig. 5 for the nongettered mc-Si sample with $N_{A}=1.5$ $\times 10^{16} \mathrm{~cm}^{-3}$. By assuming the initial $\mathrm{Fe}_{i}$ proportion to be $92 \%$ for the thermal dissociation case, the proportion of $\mathrm{Fe}_{i}$ in the case of optical dissociation $\left(5 \mathrm{~min}\right.$ of $0.1 \mathrm{~W} \mathrm{~cm}^{-2}$ white light) for the same sample is found to be, using Eq. (4), within $1 \%$ of $100 \%$.

We can now be confident that, under the optical dissociation conditions used in these experiments, almost $100 \%$ of the $\mathrm{Fe}$ is paired during the first measurement, and $100 \%$ interstitial afterwards. The appropriate value of $C$ can then be found for a given dopant density and excess carrier density, and finally, $[\mathrm{Fe}]_{\text {total }}$ can be calculated.

\section{E. Determination of Fe concentration}

In practice, it should only be necessary to measure the change in lifetime at a single value of $\Delta n$. In order to check the reliability of the technique however, we have calculated $C$ and then $[\mathrm{Fe}]_{\text {total }}$ at each value of $\Delta n$ for the partially gettered sample in Fig. 4, as well as for a neighboring wafer that was not gettered. The results are shown in Fig. 7. 
The uncertainties in $[\mathrm{Fe}]_{\text {total }}$ have been calculated by assuming a $10 \%$ uncertainty in each lifetime measurement. If the difference between the two lifetimes at a given injection level is small, the resulting uncertainty in $[\mathrm{Fe}]_{\text {total }}$ is increased. Naturally, this occurs for data near the crossover point. Nevertheless, the calculated value of $[\mathrm{Fe}]_{\mathrm{total}}$ is, as it should be, independent of $\Delta n$ within the uncertainty limits. The range of $\Delta n$ is smaller for the nongettered sample due to trapping effects obscuring the data at lower injection levels.

The concentrations of $\mathrm{Fe}$ for the gettered and nongettered wafers were determined to be $3 \times 10^{11}$ and 9 $\times 10^{11} \mathrm{~cm}^{-3}$, respectively. This reduction in $\mathrm{Fe}$ is not as large as might be hoped for when using phosphorus gettering. It may be that the gettering step is too short to allow complete gettering from deep within the wafer. Alternatively, the slow dissolution of precipitates in the wafer bulk may act as a constant source of interstitial $\mathrm{Fe}$, maintaining a certain background level despite the ongoing gettering action.

It should be emphasized that the calculated Fe concentrations in fact represent a form of area-averaged values, because the QSSPC method involves measuring a few square centimeters simultaneously, which almost always contains several grains. It is therefore likely that, in reality, the lifetime varies somewhat within this area, as may the "background" lifetime $\tau_{\text {other }}$ [in Eq. (4)]. These variations can cause distortions in the determination of $[\mathrm{Fe}]_{\text {total }}$, since the QSSPC technique gives extra weight to highphotoconductance regions. For example, if $50 \%$ of the grains have $\tau_{\text {other }}=10 \mu \mathrm{s}$ and $50 \%$ have $\tau_{\text {other }}=100 \mu \mathrm{s}$, but both contain $[\mathrm{Fe}]_{\text {total }}=5 \times 10^{11} \mathrm{~cm}^{-3}$, the resulting iron concentration will be overestimated by approximately $40 \%$. However, if the intergrain variations are not large, or the area of poor grains is small, as was the case in the regions chosen in this study, these effects will be relatively unimportant.

A more precise mapping of $[\mathrm{Fe}]_{\text {total }}$ would be possible by using a technique such as carrier density imaging. ${ }^{24}$ This steady-state technique obtains a high-resolution map of $\Delta n$ values, and therefore $\tau_{\text {eff }}$, across a sample at a fixed generation rate. If the lifetime at a given position on the wafer changes during dissociation, and the same generation rate is used for both measurements, the resulting $\Delta n$ values will be different. By contrast, when using the QSSPC technique, which sweeps across a range of generation rates, the lifetimes before and after dissociation can be measured at the same average $\Delta n$ value (although, of course, this will vary somewhat between grains). Using a fixed generation technique would therefore require a slight modification in the calculation of $C$. Since the iron concentration and lifetime may also vary laterally across a sample, such a modified $C$ value should, in principle, be calculated for every point.

Other differential lifetime mapping techniques, such as microwave-detected photoconductance decay, may not be as well suited for this approach to Fe mapping. This is because they do not directly measure the value of $\Delta n$, but can only infer it from the measured lifetime value by assuming the lifetime is injection independent. ${ }^{25}$ This may especially cause problems when measuring the lifetime after dissociation, since then the injection dependence is quite pronounced.

\section{CONCLUSIONS}

Existing procedures for measuring Fe concentrations using low-injection SPV diffusion length measurements on 5-15 $\Omega \mathrm{cm}$ Si have been extended to include arbitrary doping, as well as mid- and high-injection lifetime measurements. This allows the use of fast, contactless lifetime techniques for very sensitive measurement of $\mathrm{Fe}$ concentrations on any crystalline silicon material. This extended technique is slightly more complex than the SPV approach, since the prefactor for determining $[\mathrm{Fe}]_{\text {total }}$ changes with the injection level, which may, of course, vary across a sample due to changing levels of $\mathrm{Fe}$.

In addition, a characteristic fingerprint of $\mathrm{Fe}$ contamination has been presented. The crossover point between the lifetime curves before and after dissociation always occurs in the range from 1.4 to $2.0 \times 10^{14} \mathrm{~cm}^{-3}$ for any doping density below $5 \times 10^{16} \mathrm{~cm}^{-3}$. This provides a fast and convenient diagnostic tool for Fe contamination.

\section{APPENDIX A}

The condition for the lifetime to be equal before and after dissociation is

$$
\frac{1}{\tau_{\mathrm{FeB}}\left(N_{1}\right)}+\frac{1}{\tau_{\mathrm{Fe}_{i}}\left(N_{2}\right)}=\frac{1}{\tau_{\mathrm{FeB}\left(N_{3}\right)}}+\frac{1}{\tau_{\mathrm{Fe}_{i}}\left(N_{4}\right)} .
$$

The parameters $N_{1}$, etc. represent the density of recombination centers, so that $N_{1}+N_{2}=N_{3}+N_{4}=[\mathrm{Fe}]_{\text {total }}$.

From the linear nature of the SRH model, lifetimes caused by different concentrations of the same recombination center are related by

$$
\tau_{\mathrm{FeB}}\left(N_{1}\right)=\frac{N_{2}}{N_{1}} \tau_{\mathrm{FeB}}\left(N_{2}\right) .
$$

Using this fact, we may then re-write Eq. (A1), after some rearranging, as

$$
\frac{1}{\tau_{\mathrm{FeB}}\left(N_{1}\right)}=\frac{1}{\tau_{\mathrm{Fe}_{i}}\left(N_{2}\right)}\left(\frac{N_{4}-N_{2}}{N_{2}}\right)\left(\frac{N_{1}}{N_{1}-N_{3}}\right) .
$$

Making use of the fact that $N_{4}-N_{2}=N_{1}-N_{3}$, and then applying an analog of Eq. (A2), this reduces to the desired result:

$$
\tau_{\mathrm{FeB}}\left(N_{1}\right)=\tau_{\mathrm{Fe}_{i}}\left(N_{1}\right) .
$$

This result is quite general, and would also apply to other impurities that undergo some type of change of state that leads to a crossover in the injection dependent lifetime curves. It should be noted, however, that some pairs of metastable impurities (for e.g., $\mathrm{CrB}$ and $\mathrm{Cr}_{i}$ ), do not exhibit such a crossover, at least for some dopant densities. ${ }^{8}$ For such sets of energy levels and cross sections, there exists no value of $\Delta n$ that satisfies Eq. (A4).

\section{ACKNOWLEDGMENTS}

This work has been supported by NOVEM (The Netherlands Agency for Energy and the Environment) under contract no. 2020.01.13.11.2002. The authors are grateful to A. 
Cuevas, J. Bultman, and A. Weber for helpful discussions, and to staff of ECN for assistance with sample preparation.

${ }^{1}$ G. Zoth and W. Bergholz, J. Appl. Phys. 67, 6764 (1990).

${ }^{2}$ J. Lagowski, P. Edelman, A. M. Kontkiewicz, O. Milic, W. Henley, M. Dexter, L. Jastrzebski, and A. M. Hoff, Appl. Phys. Lett. 63, 3043 (1993).

${ }^{3}$ L. Jastrzebski, W. Henley, D. Schielein, and J. Lagowski, J. Electrochem. Soc. 142, 3869 (1995)

${ }^{4}$ W. B. Henley and D. A. Ramappa, J. Appl. Phys. 82, 589 (1997).

${ }^{5}$ R. A. Sinton and A. Cuevas, Appl. Phys. Lett. 69, 2510 (1996).

${ }^{6}$ D. Macdonald and A. Cuevas, Appl. Phys. Lett. 74, 1710 (1999).

${ }^{7}$ D. Macdonald, A. Cuevas, and J. Wong-Leung, J. Appl. Phys. 89, 7932 (2001).

${ }^{8}$ T. Pavelka, in Recombination Lifetime Measurements in Silicon, edited by D. C. Gupta, F. R. Bacher, and W. M. Hughes (American Society for Testing and Materials, Philadelphia, 1998), p. 206.

${ }^{9}$ C. Ballif, S. Peters, D. Borchert, C. Hassler, J. Isenberg, R. Schindler, W. Warta, and G. Willeke, Proceedings of the 17th EC-PVSEC, edited by B. McNelis, W. Palz, H. A. Ossenbrink, and P. Helm (WIP, Munich, 2001).

${ }^{10} \mathrm{R}$. Falster and G. Barionetti, in Recombination Lifetime Measurements in Silicon, edited by D. C. Gupta, F. R. Bacher, and W. M. Hughes (American Society for Testing and Materials, Philadelphia, 1998), p. 226.

${ }^{11}$ W. Shockley and W. T. Read, Phys. Rev. 87, 835 (1952).
${ }^{12}$ R. N. Hall, Phys. Rev. 87, 387 (1952).

${ }^{13}$ J. S. Blakemore, Semiconductor Statistics, International Series of Monographs on Semiconductors Vol. 3 (Pergamon, Oxford, 1962).

${ }^{14}$ W. M. Bullis and H. R. Huff, J. Electrochem. Soc. 143, 1399 (1996).

${ }^{15}$ A. A. Istratov, H. Hieslmair, and E. R. Weber, Appl. Phys. A: Mater. Sci. Process. 69, 13 (1999).

${ }^{16}$ P. A. Basore and D. A. Clugston, PCID v5.5 (University of New South Wales, Sydney, Australia, 2000).

${ }^{17}$ L. C. Kimerling and J. L. Benton, Physica B 116, 297 (1983).

${ }^{18}$ A. G. Aberle, Crystalline Silicon Solar Cells: Advanced Surface Passivation and Analysis (Center for Photovoltaic Engineering, University of New South Wales, Sydney, Australia, 1999).

${ }^{19}$ J. Schmidt and A. Cuevas, J. Appl. Phys. 86, 3175 (1999)

${ }^{20}$ W. B. Henley, D. A. Ramappa, and L. Jastrezbski, Appl. Phys. Lett. 74, 278 (1999).

${ }^{21}$ S. A. McHugo, A. Thompson, I. Perichaud, and S. Martinuzzi, Appl. Phys. Lett. 72, 3482 (1998).

${ }^{22}$ M. J. Kerr and A. Cuevas, Semicond. Sci. Technol. 17, 166 (2002).

${ }^{23}$ D. Macdonald, R. A. Sinton, and A. Cuevas, J. Appl. Phys. 89, 2772 (2001).

${ }^{24}$ J. Isenberg, S. Riepe, S. W. Glunz, and W. Warta, J. Appl. Phys. 93, 4268 (2003).

${ }^{25}$ J. Schmidt, IEEE Trans. Electron Devices 46, 2018 (1999). 